\title{
Screening for Carotid Artery Stenosis and Performance of Carotid Endarterectomy in Select Asymptomatic Patients is Cost-effective
}

\author{
Filiberto Rodriguez $^{* \dagger}$, B.A., M.D.
}

\section{INTRODUCTION}

Stroke (acute neurological dysfunction lasting more than 24 hours caused by disturbance of the cerebral blood supply) is the third leading cause of death in the United States after myocardial infarction and cancer (1); see Box 1). The overall incidence of stroke is approximately one per 1000 people in the general population, but reaches 20 per 1000 people in the octogenarian population and in all age groups is slightly higher among males (2). Intracerebral and subarachnoid hemorrhages account for only $20 \%$ of strokes while $80 \%$ of strokes are of the ischemic type (2; see Box 1). There is a $2-5 \%$ annual ipsilateral stroke risk for people with internal carotid artery (ICA) stenosis of greater than 50\% diameter reduction (3-10). Approximately $0.5 \%$ of people in their $50 \mathrm{~s}$ and about $10 \%$ of those over age 80 have carotid artery stenosis $>50 \%$ (11).

Half of all strokes are minor, with complete resolution of symptoms within three months (12). In contrast, almost $20 \%$ of people that suffer a major stroke die in the first week and 33\% do not survive the first year (2). The remaining two-thirds of patients who survive a major stroke experience permanent physical and mental disabilities and have a threefold-increased risk for recurrent ipsilateral stroke $(2,12)$. The projected life expectancy for people after major stroke is approximately six years, with a projected cost of $\$ 151,000$ (all funds in \$US) (12). Aggregate life-time costs associated with stroke in the United States total

\footnotetext{
*To whom correspondence should be addressed: Filiberto Rodriguez, 4250 El Camino Real A-109, Palo Alto, California 943061; filrodriguez@hotmail.com

†University of Virginia School of Medicine, Charlottesville, Virginia, USA
}

over $\$ 40$ billion with major costs attributable to chronic nursing home and home-health care needs of patients disabled by stroke $(1,12)$. The economic impact of stroke is similarly high in the Canadian context (13).

Carotid endarterectomy (CEA) is a surgical excision of stenosed sections of the carotid artery (see Box 1). Prophylactic CEA to reduce the incidence of stroke in asymptomatic patients with high-grade carotid artery stenosis (> 60\%) has been recommended by both the American Heart Association and the Joint Council of the Society for Vascular Surgery and the North American Chapter of the International Society for Vascular Surgery $(3,14)$. It remains uncertain, however, whether the benefits of prophylactic endarterectomy warrant the associated expenses and risks of widespread screening.

This article first reviews the literature showing that CEA for asymptomatic people with significant ICA stenosis is a beneficial and cost-effective treatment modality. Having shown that there is an effective method of prophylaxis for people at risk of stroke, the major question remaining is how to screen the population to identify those people who should receive the treatment. These issues are examined within a costeffectiveness framework in order to establish a rational protocol for selectively screening certain subpopulations to identify those individuals who would most benefit from prophylactic CEA.

\section{CAROTID ENDARTERECTOMY}

\section{Benefits of CEA for the Patient}

In 1991, the North American Symptomatic Carotid Endarterectomy Trial (NASCET) showed that CEA is 
Box 1. Pathology and treatment of stroke

The left and right internal carotid arteries are the major blood supply to the anterior and middle cerebral arteries. Occlusion of the carotid arteries can therefore cause cerebrovascular insufficiency and stroke. Stenotic atherosclerotic plaques, emboli, or both may cause the occlusion. Atherosclerosis is particularly common at the bifurcation of the carotid artery (i.e., carotid bulb or sinus) in the neck, where blood flow is turbulent (1). Disruption of the cerebral blood supply may result in transient ischemic attacks, longer lasting reversible ischemic neurologic deficits, or permanent damage by cerebral infarction (i.e., stroke) depending on the degree and duration of the reduced blood supply.

Control of risk factors (e.g., hypertension), anticoagulation with heparin and warfarin, and administration of antiplatelet drugs (e.g., aspirin) constitute the medical therapy of cerebrovascular disease. The specific surgical procedure used to treat stenosed carotid arteries in order to reduce the risk of stroke is named carotid endarterectomy. This procedure involves the excision of the diseased artery wall, generally at the bifurcation of the common carotid artery into the internal and external branches. The endolthelium and occluding plaque are removed, along with a portion of the media. For more detailed information on carotid endarterectomy see references (2-4).

\section{REFERENCES}

1. Moore WS, Barnett HJM, Beebe HG, et al. Guidelines for carotid endarterectomy: a multidiciplinary consensus from the Ad Hoc Committee, American Heart Association. Stroke 26: 188-201; 1995.

2. Morris PJ. Carotid Artery. In: Morris PJ, Malt RA, editors. Oxford Textbook of Surgery vol. 1. New York: Oxford University Press; 1994: 401-408

3. Messina LM, Zelenock GB. Cerebrovascular occlusive disease. In: Greenfield LJ, Mulholland M, Oldham KT, et al., editors. Surgery: Scientific Principles and Practice. New York: Lippincott-Raven; 1997: 1745-1758

4. Gewertz BL, Graham A, Lawrence P, et al. Diseases of the vascular system. In: Lawrence PF, editor. Essentials of General Surgery. Philadelphia: Williams and Wilkins; 1992: 328-347.

beneficial in symptomatic patients with ICA stenosis of greater than $70 \%$ (15). The NASCET demonstrated a $65 \%$ reduction in the relative risk of ipsilateral stroke with CEA in symptomatic patients and a $17 \%$ reduction in absolute risk after two years $(p<0.001)$. As a result, CEA for symptomatic patients with high-grade carotid artery stenosis has become widely accepted in clinical practice (12).

The Asymptomatic Carotid Atherosclerosis Study (ACAS) similarly provided evidence that asymptomatic patients with high-grade stenosis also benefit from surgical rather than medical management (4). Several earlier studies, including the European Carotid Artery with Asymptomatic Narrowing: Operation Versus Aspirin (CASANOVA) trial and the U.S. veterans trial, had suggested an advantage of surgical over medical therapy for asymptomatic carotid artery stenosis prior to the ACAS, but these studies lacked statistical power and generalizability $(6,9,16,17)$. The prospective, multicenter ACAS randomized 1662 patients with asymptomatic ICA stenosis between $60 \%$ and $99 \%$ (mean stenosis $73 \%$ ) to CEA plus aspirin or aspirin alone. This study demonstrated a 54\% reduction in relative risk of ipsilateral stroke from $2.3 \%$ per year under medical management to $1 \%$ per year after CEA ( $p$ $=0.004)(4)$.

The 54\% relative risk reduction for ipsilateral stroke in the ACAS approximates the $65 \%$ relative risk reduction for stroke in symptomatic patients after CEA in the NASCET. Unlike the NASCET, however, which demonstrated a $17 \%$ reduction in absolute risk of stroke after two years, the ACAS demonstrated that asymptomatic patients have only a $6 \%$ reduction in absolute risk (from $11 \%$ to $5.1 \%$ ) five years after surgery compared with medically managed patients $(4,15)$. This difference is attributable to the greater likelihood of stroke during medical management of symptomatic patients (13\% per year in NASCET) compared with asymptomatic patients $(2.3 \%$ per year in ACAS) (12). The ACAS authors noted that 19 CEAs would have to be performed to prevent one stroke in five years. Moreover, the ACAS results were based on results from medical centers and surgeons with documented perioperative morbidity less then 3\% (4). Although the ACAS successfully demonstrated a statistically significant advantage for surgical prophylaxis of stroke from asymptomatic ICA stenosis of greater than $60 \%$, the cost-effectiveness of prophylactic CEA must be assessed.

\section{Cost-Effectiveness of CEA for Asymptomatic Stenosis}

The literature shows that endarterectomy for asymptomatic carotid artery stenosis is cost-effective $(12,18,19)$. The cost-effectiveness of CEA for asymptomatic ICA stenosis of greater than $60 \%$ can be determined by calculating a cost-effectiveness ratio in dollars/quality-adjusted life year saved (QALY). Cronenwett et al. used a Markov decision model to perform such a cost-effectiveness analysis (12). This model compares surgical versus medical treatment by scoring patient outcomes from 0 (death) to 1 (alive without impairment). Data from clinical trials are used to assign probabilities for these outcomes. This information is then translated into the incremental cost per QALY by surgical treatment compared with medical management. A low cost-effectiveness ratio (dollars/QALY) indicates a cost-effective therapy (12).

The majority of costs in the medical group are associated with stroke, while most costs in the surgical 
Table 1. Cost-effectiveness of selected medical practices (from reference (12)).

\begin{tabular}{lc}
\hline \hline Medical Practice & Cost/QALY (1996 U.S. Dollars) \\
\hline Treatment of mild-moderate hypertension compared with no treatment & $\$ 13,000$ \\
Propranolol & $\$ 87,000$ \\
Captopril & $\$ 53,000$ \\
Hemodialysis for end-stage renal disease & $\$ 4,600$ \\
Total hip replacement for severe arthritis & $\$ 7,000$ \\
Coronary artery bypass compared to medical treatment of severe angina & $\$ 51,000$ \\
Left main disease & $\$ 33,000$ \\
Single-vessel disease & $\$ 20,000$ \\
Transplantation compared with medical treatment & $\$ 189,000$ \\
Heart & $\$ 770,000$ \\
Kidney & $\$ 8,000$ \\
Treatment of hyperlipidemia with cholestyramine & \\
Universal precautions for HIV precautions in health-care workers & \\
CEA for asymptomatic ICA stenosis $>60 \%$ & \\
\hline \hline
\end{tabular}

QALY: quality-adjusted life year saved

group are associated with the initial costs of endarterectomy. Stroke is estimated to cost $\$ 34,000$ for care during the first year plus $\$ 18,000$ for each additional year of life after the stroke (12). Seventy-nine percent of total costs associated with the medical management of asymptomatic patients with high-grade carotid artery stenosis are from the initial and subsequent care of patients who suffer a major stroke despite treatment. In addition, $26 \%$ of medically managed patients become symptomatic and develop transient ischemic attacks or suffer minor strokes. Endarterectomy for these newly symptomatic patients diminishes the cost-effectiveness of medical management and comprises $15 \%$ of the total costs for this group (12). In the surgical group, $67 \%$ of total costs are associated with initial CEA. The average cost of this procedure is $\$ 8,500$. Thirty one percent of the costs in the surgical group are from the care of patients who suffer major stroke despite prophylactic CEA.

Using this data, medical treatment has been found to provide a projected quality-adjusted life expectancy of 7.87 QALYs versus 8.12 QALYs after surgical treatment, a difference of 0.25 QALYs (three months) in favor of surgical treatment (12). The predicted lifetime (discounted) cost was $\$ 12,407$ for medical and $\$ 14,448$ for surgical treatment, a difference of $\$ 2,041$ in favor of medical treatment. Endarterectomy for asymptomatic ICA stenosis of greater than $60 \%$ costs $\$ 2,041$ more per patient than medical management alone, but 0.25 QALYs are gained for each patient treated with CEA. Hence, the incremental cost-effectiveness ratio for surgical treatment of asymptomatic carotid artery stenosis of greater than $60 \%$ is $\$ 8,004$ per QALY (\$2041/0.25 QALYs).

The cost-effectiveness ratio of prophylactic CEA for asymptomatic patients with high-grade ICA stenosis
(\$8,004/QALY) compares favorably with other commonly accepted medical practices. In general, medical technologies that cost less than $\$ 20,000 /$ QALY are accepted as appropriate expenditures of societal resources (16). Coronary artery bypass (CAB), for example, has a cost-effectiveness ratio of \$7,000/QALY for left main disease and \$51,000/QALY for singlevessel disease compared to medical treatment of severe angina (12). In addition, many technologies that cost between $\$ 20,000$ and $\$ 100,000 / \mathrm{QALY}$ are commonly provided. Table 1 lists the cost-effectiveness ratios for several accepted medical practices. Comparison of the calculated cost of CEA to Table 1 demonstrate that CEA for asymptomatic ICA stenosis of greater than $60 \%$ is cost-effective and suggest that prophylactic endarterectomy should be accepted in clinical practice as an appropriate treatment modality for asymptomatic patients with significant carotid artery stenosis. The question remaining is how to identify (in a costeffective manner) those patients who would most benefit from the treatment.

\section{SCREENING FOR CAROTID ARTERY STENOSIS}

\section{Risk Factors for Stroke}

Persons with asymptomatic carotid artery stenosis have an increased risk of stroke (3-11,20-23). In addition to a history of carotid artery disease, the principal risk factors for ischemic stroke are increased age, hypertension, smoking, coronary artery disease, atrial fibrillation, and diabetes $(11,24-27)$. Of these, the most important modifiable risk factors are hypertension and smoking $(11,28)$. Improved treatment of high blood pressure has been credited with the 50\% reduction in age-adjusted stroke mortality observed 
since 1972 (11,29,30). Consequently, an awareness of the diagnosis of high-grade carotid artery stenosis and its associated risks may motivate asymptomatic patients to modify their risk factors. Furthermore, this information helps identify those people who might benefit most from screening.

\section{Methods of Screening}

The rationale for screening for carotid artery stenosis is that early detection and treatment (including modification of risk factors) can reduce morbidity due to cerebrovascular disease. Two methods are used to screen for carotid artery stenosis: clinical auscultation for carotid bruits and noninvasive studies of the carotid arteries.

Neck auscultation is an imperfect screening test for carotid artery stenosis (11). It is problematic because of the considerable inter-observer variation among clinicians in the interpretation of the intensity, pitch, and duration of the bruit heard (31). In addition, a cervical bruit can be heard in $4 \%$ of the population over age 40 , but the finding is not specific for significant carotid artery stenosis (11). Between $40 \%$ and $75 \%$ of arteries with bruits do not have significant compromise in blood flow (32); similar sounds can be produced by anatomic variation, tortuosity, venous hum, goiter, and transmitted cardiac murmurs $(11,16,31,33,34)$. Moreover, hemodynamically significant stenotic lesions may exist in the absence of an audible bruit $(31,33,35)$. Using 70-99\% stenosis on carotid angiogram as a reference standard, auscultation of a carotid bruit has been found to have a sensitivity of only $63-76 \%$ and a specificity of only $61-76 \%$ for clinically significant stenosis $(11,36)$. The positive predictive value (PPV) for auscultation of a carotid bruit has been found to be only $34 \%$ (37).

Patients with cervical bruits, however, can be evaluated further with greater accuracy by noninvasive study of the carotid arteries. Duplex ultrasound combines the capabilities of B-mode and Doppler ultrasound and costs $\$ 150$ (11). Compared with carotid contrast angiography (the reference standard), carotid duplex ultrasound has a sensitivity of $96 \%$ and specificity of 66\% (38). Depending on the underlying population characteristics, the PPV of duplex ultrasound ranges from $82 \%$ to $97 \%$ (39). Magnetic resonance arteriography (MRA) is a newer imaging technique, which provides $100 \%$ sensitivity and $76 \%$ specificity compared with contrast angiography (38). However, MRA costs over $\$ 400$ which precludes using it as a screening modality (11).

Since asymptomatic carotid artery stenosis can present as major stroke, it may be possible to prevent stroke by screening for asymptomatic carotid artery disease. Although auscultation of the carotid arteries is widely considered a routine component of the physical examination, the Canadian Task Force on the Periodic Health Examination has recommended against auscultation for screening based on the poor sensitivity and specificity of cervical bruits as an indicator of significant carotid artery stenosis (41). Widespread screening with duplex ultrasound in the primary care setting may be an effective way to reduce morbidity and mortality from stroke. Statistically, the patients most likely to benefit from screening are men over the age 60 who have other risk factors for stroke, no contraindications to major surgery, and access to highquality vascular surgeons (11).

Annual ultrasonography is not needed to determine the risk of stroke in patients with asymptomatic carotid artery disease $(7,40)$. The baseline degree of carotid artery stenosis on initial duplex ultrasound is the most significant predictor of future stroke, and it retains its predictive power for more than three years (40). Hence, asymptomatic patients with baseline ICA stenosis of greater than $60 \%$ can be considered for surgery without serial duplex scans. Prophylactic CEA should be considered if patients have a projected life expectancy greater than five years and the perioperative morbidity and mortality rates are less than $3 \%(4,14)$.

\section{Cost-Effectiveness of Screening}

Although the cost of screening $50 \%$ of the population over age 60 in the U.S. has been estimated to be $\$ 7$ billion (11), screening for asymptomatic carotid artery stenosis can be cost-effective. The cost-effectiveness ratio for screening 65 year old men in the general population with one-time carotid duplex ultrasounds is approximately $\$ 53,000 / \mathrm{QALY}$ (42). This ratio compares with the cost-effectiveness ratios of hemodialysis for end-stage renal disease and $\mathrm{CAB}$ for single-vessel disease (Table 1). Following patients with yearly duplex scans is not cost-effective (\$458,000/QALY) (42). Since the baseline degree of ICA stenosis is the most significant predictor of future stroke, a one-time screening program represents a rational approach for widespread screening.

The cost-effectiveness of screening for carotid artery stenosis can be enhanced by targeting a sub-population with a known high prevalence of ICA stenosis, e.g., of greater than $60 \%$. This strategy lowers the costeffectiveness ratio for one-time screening to $\$ 35,000 /$ QALY (42). Three sub-groups of patients have been identified with a high prevalence (> 20\%) of asymptomatic high-grade carotid artery stenosis: patients with claudication, lower extremity bypass (LEB) patients, and patients with coronary artery disease (43-45). Twenty-four percent of patients who 
present with claudication as their chief complaint have asymptomatic ICA stenosis of greater than 50\% (43). The prevalence of asymptomatic carotid artery stenosis in both CAB and LEB patients approaches $28 \%(44,45)$. Experience with $\mathrm{CAB}$ patients provides evidence favoring targeted screening for asymptomatic carotid artery stenosis. Preoperative screening with duplex ultrasound of $\mathrm{CAB}$ patients for concomitant carotid artery disease has been shown to reduce the incidence of stroke associated with cardiopulmonary bypass (46). Consequently, the single-stage CEA-CAB procedure has become well-accepted (47). Patients with claudication, LEB, and coronary artery disease represent a high-risk patient population in which routine screening with duplex ultrasound is justified and costeffective.

\section{Further Reducing the Cost of Imaging}

Reducing the morbidity associated with contrast angiography can further enhance the cost-effectiveness of CEA and screening for carotid artery stenosis. The cost of surgical treatment for carotid artery stenosis increases from \$630/QALY to \$14,450/QALY when perioperative mortality and morbidity rise from $0.5 \%$ to $4.2 \%$ (12). The total risk of perioperative mortality and morbidity from endarterectomy encompasses both the risk from surgery alone and the risk from contrast angiography. The risk that results from surgery alone is approximately $1.7 \%$ (12). Contrast angiography constitutes $0.6-1.2 \%$ of the total perioperative event rate $(12,19)$. Noninvasive preoperative imaging can reduce the perioperative event rate of CEA by eliminating the risk associated with contrast angiography.

Magnetic resonance arteriography and duplex ultrasound can be combined to effectively image patients noninvasively for endarterectomy (19). Compared with contrast angiography as the reference standard, MRA combined with carotid duplex ultrasound has been found to be $100 \%$ sensitive and $86 \%$ specific (19). In this series, contrast angiography was needed to confirm only $26 \%$ of all MRA/duplex results. MRA and duplex ultrasound combined also cost $\$ 1050$ less than contrast angiography $(11,12)$. The routine use of noninvasive preoperative imaging can eliminate the costs and risks associated with contrast angiography in $74 \%$ of patients imaged prior to CEA.

\section{CONCLUSION}

The proportion of all strokes attributable to previously asymptomatic carotid artery stenosis has been estimated to be $13 \%$ (23). This comprises $\$ 5.2$ billion of the $\$ 40$ billion associated with stroke spent annually in the United States. In addition, the disability that results from major stroke imposes enormous emotional burdens on family members and caregivers, which cannot be expressed in monetary terms. Widespread screening for asymptomatic carotid artery stenosis could cost approximately $\$ 7$ billion. This cost may be reduced by targeting screening efforts to a subpopulation of patients at increased risk for severe carotid artery disease. The cost-effectiveness of this strategy is estimated at $\$ 35,000 /$ QALY. Endarterectomy for those patients found to have asymptomatic carotid artery stenosis of greater than $60 \%$ must be included in the total cost analysis. Since the cost-effectiveness of CEA is approximately $\$ 8000 / \mathrm{QALY}$, the cumulative cost-effectiveness of targeted screening and surgery for high-grade carotid artery stenosis can be estimated to be $\$ 43,000 /$ QALY.

Carotid endarterectomy for asymptomatic carotid artery disease is clearly cost effective when compared with medical treatment alone. Screening for carotid artery stenosis in asymptomatic high-risk patients is also cost effective. However, the most effective interventions to prevent stroke are smoking cessation and aggressive treatment of hypertension. Although widespread screening could detect some patients with high-grade carotid artery lesions who may benefit from surgical intervention, such patients ultimately account for only a small proportion of all strokes. The risk of major stroke ipsilateral to asymptomatic stenotic lesions is relatively low at $1 \%$ per year (11). In addition, widespread screening of the general population with carotid ultrasound will subject some patients without significant carotid artery disease to the risks of contrast angiography, which carries a $1 \%$ risk of stroke, due to occasional false-positive results of duplex ultrasound (11).

Despite the $54 \%$ reduction in relative-risk for ipsilateral stroke in asymptomatic patients with ICA stenosis of greater than $60 \%$, the ACAS authors noted that 19 CEAs would have to be performed with surgical morbidity and mortality less than $3 \%$ to prevent one stroke in five years (4). Carotid endarterectomy is, therefore, effective in reducing the chance of stroke in certain people, but the challenge is to efficiently identify those people who would benefit from treatment. It seems that widespread screening of the general population in the primary care setting is not a cost-effective way to reduce the morbidity and mortality of stroke. Instead, the potential benefits of screening should be discussed with high-risk patients and certain sub-populations of high-risk patients should be screened on a routine basis. Selective screening and prophylactic CEA for patients at risk of stroke should therefore be included in our attempts to reduce the incidence of stroke. 


\section{REFERENCES}

1. Elkind MS and Sacco RL. Stroke risk factors and stroke prevention. Seminars in Neurology 18: 429-440; 1998.

2. Horst GJ and Postigio A. Stroke: prevalence and mechanisms of cell death. In: Horst GJ and Korf J, eds. Clinical Pharmacology of Cerebral Ischemia. Towata, NJ: Humana Press; 1997: 1-30.

3. Moore WS, Barnett HJM, Beebe HG, et al. Guidelines for carotid endarterectomy: a multidiciplinary consensus from the Ad Hoc Committee, American Heart Association. Stroke 26: 188-201; 1995.

4. Executive Committee for the Asymptomatic Carotid Atherosclerosis Study. Endarterectomy for asymptomatic carotid artery stenosis. JAMA 273: 1421-1428; 1995.

5. European Carotid Surgery Trialists' Collaborative Group. Risk of stroke in the distribution of an asymptomatic carotid artery. Lancet 345: 209-212; 1995.

6. Hobson RW II, Weiss DG, Fields WS, et al. Efficacy of carotid endarterectomy for asymptomatic carotid stenosis. New England Journal of Medicine 328: 221-227; 1993.

7. Mansour MA, Mattos MA, Faught WE, et al. The natural history of moderate $(50 \%$ to $79 \%)$ internal carotid artery stenosis in symptomatic, nonhemispheric, and asymptomatic patients. Journal of Vascular Surgery 21: 346-358; 1995.

8. Norris JW, Zhu CZ, Bornstein NM, et al. Vascular risks of asymptomatic carotid stenosis. Stroke 22: 1485-1490; 1991.

9. The CASANOVA Study Group. Carotid surgery versus medical therapy in asymptomatic carotid stenosis. Stroke 22: 1229-1235; 1991.

10. Ellis MR, Franks PJ, Cuming R, et al. Prevalence, progression and natural history of asymptomatic carotid stenosis: is there a place for carotid endarterectomy? European Journal of Vascular Surgery 6: 172-177; 1992.

11. Tabet S, Berg AO, Atkins D. Screening for asymptomatic carotid stenosis. Guide to clinical preventive services, Report of the U.S. Preventive Services Task Force, 2nd Edition. Baltimore, MA: Williams and Wilkins; 1996: 53-61.

12. Cronenwett JL, Birkmeyer JD, Nackman GB, et al. Costeffectiveness of carotid endarterectomy in asymptomatic patients. Journal of Vascular Surgery 25: 298-309; 1997.

13. Chan B and Hayes B. Cost of stroke in Ontario, 1994/95. Canadian Medical Association Journal 159: S2-8; 1998.

14. Moore WS, Moh JP, Najafi H, et al. Carotid endarterectomy: practice guidelines. Journal of Vascular Surgery 15: 469-479; 1992.

15. North American Symptomatic Carotid Endarterectomy Trial Collaborators. Beneficial effect of carotid endarterectomy in symptomatic patients with high-grade carotid stenosis. New England Journal of Medicine 325: 445-453; 1991.

16. Thompson JE, Patman RD and Talkington CM. Asymptomatic carotid bruit: long-term outcome of patients having endarterectomy compared with unoperated controls. Annals of Surgery 188: 308-316; 1978.

17. Moneta GL, Taylor DC, Nicholls SC, et al. Operative versus nonoperative management of asymptomatic high-grade internal carotid artery stenosis: improved results with endarterectomy. Stroke 18: 1005-1010; 1987.

18. Kuntz KM and Kent KC. Is carotid endarterectomy costeffective? An analysis of symptomatic and asymptomatic patients. Circulation 94 (Supplement 9): 194-198; 1996.

19. Kuntz KM, Skillman JJ, Whittemore AD, et al. Carotid endarterectomy in asymptomatic patients: is contrast angiography necessary? A morbidity analysis. Journal of Vascular Surgery 22: 706-714; 1995.

20. Heyman A, Wilkinson WE, Heyden S, et al. Risk of stroke in asymptomatic persons with cervical arterial bruits: a population study in Evans County, Georgia. New England Journal of Medicine 302: 838-841; 1980.

21. Wolf PA, Kannel WB, Sorlie P, et al. Asymptomatic carotid bruit and risk of stroke: the Framingham study. JAMA 245: 1442$1445 ; 1981$

22. Warlow C. Endarterectomy for asymptomatic stenosis? Lancet 345: 1254-1255; 1995.

23. Amarenco P, Cohen A, Tzourio C, et al. Atherosclerotic disease of the aortic arch and the risk of ischemic stroke. New England Journal of Medicine 331: 1474-1479; 1994.

24. Matchar DB, McCrory DC, Barnett HJM, et al. Medical treatment for stroke prevention. Annals of Internal Medicine 121: 41-53; 1994.

25. Schoenberg BS. Epidemiology of cerebrovascular disease. Southern Medical Journal 72: 331-336; 1979.

26. Davis PH, Dambrosa JM, Schoenberg DG, et al. Risk factors for ischemic stroke: a prospective study in Rochester, Minnesota. Annals of Neurology 22: 319-327; 1987.

27. D'Agostino RB, Wolf PA, Belanger AJ, et al. Stroke risk profile: adjustment for antihypertensive medication. The Framingham Study. Stroke 25: 40-43; 1994.

28. Whisnant JP, Homer D, Ingall TJ, et al. Duration of cigarette smoking is the strongest predictor of severe extracranial carotid artery atherosclerosis. Stroke 21: 707-714; 1990.

29. Collins R, Peto R, MacMahon S, et al. Blood pressure, stroke, and coronary artery disease: part 2, short-term reductions in blood pressure: overview of randomized drug trials in their epidemiological context. Lancet 335: 827-838; 1990.

30. Medical Research Council Working Party. Persistence of reduction in blood pressure and mortality of participants in the hypertension detection and follow-up program. JAMA 259: 2113-2122; 1988

31. Chambers BR, Norris JW. Clinical significance of asymptomatic neck bruits. Neurology 35: 742-745; 1985.

32. Quinones-Baldrich WJ, Moore WS. Asymptomatic carotid stenosis: rationale for management. Archives of Neurology 42: 378-382; 1985.

33. Caplan LR. Carotid artery disease. New England Journal of Medicine 315: 886-888; 1986.

34. Chambers BR, Norris JW. Outcome in patients with asymptomatic neck bruits. New England Journal of Medicine 315: 860-865; 1986.

35. Kuller LH, Sutton KC. Carotid artery bruit: is it safe and effective to auscultate the neck? Stroke 15: 944-47; 1984.

36. Sauve JS, Laupacis A, Ostbye T, et al. Does this patient have a clinically important carotid bruit? JAMA 270: 2843-2845; 1993.

37 Davies KN, Humphrey PR. Do carotid bruits predict disease of the internal carotid arteries? Postgraduate Medical Journal 70: 433-435; 1994.

38. Kuntz KM, Skillman JJ, Whittemore AD, Kent KC. Carotid endarterectomy in asymptomatic patients: is contrast angiography necessary? A morbidity analysis. Journal of Vascular Surgery 22: 706-714; 1995.

39. Zwiebel WJ. Duplex sonography of the cerebral arteries: efficacy, limitations, and indications. American Journal of Radiology 158: 29-36; 1992.

40. Lewis RF, Abrahamowics M, Cote R, et al. Predictive power of duplex ultrasonography in asymptomatic carotid disease. Annals of Internal Medicine 127: 13-20; 1997.

41. Canadian Task Force on the Periodic Health Examination. Canadian guide to clinical preventive health care. Ottawa, Ont: Canadian Communication Group 90; 1994; 1679-1687.

42. Derdeyn CP and Powers WJ. Cost-effectiveness of screening for asymptomatic carotid atherosclerotic disease. Stroke 27: 1944- 
1950; 1996.

43. Marek J, Mills JL, Harvich J, et al. The utility of routine carotid duplex screening in patients who have claudication. Journal of Vascular Surgery 24: 572-577; 1996.

44. Gentile AT, Taylor LM Jr., Moneta GL, et al. Prevalence of asymptomatic carotid stenosis in patients undergoing infrainguinal bypass surgery. Archives of Surgery 130: 900-904; 1995.

45. Sanguigni V, Gallu M and Strano A. Incidence of carotid artery atherosclerosis in patients with coronary artery disease. Angiology 44: 34-38; 1993.

46. Pillai L, Gutierrez IZ, Curl GR, et al. Evaluation and treatment of carotid stenosis in open-heart surgery patients. Journal of Surgical Research 57: 312-315; 1994.

47. Di Tommaso L, Caputo M, Ascione R, et al. Carotid endarterectomy and myocardial revascularization. A single stage procedure. Minerva Cardioangiologica 43: 469-474; 1995.

Filiberto Rodriguez received his B.A. in 1995 from Princeton University (Princeton, New Jersey, USA) where he majored in economics with politics and Latin American studies. He completed his medical degree in 1999 at the University of Virginia School of Medicine (Charlottesville, Virginia, USA) where he conducted research in stroke and neuroprotection. Dr. Rodriguez has recently begun his post-graduate training at Standford University (Stanford, California, USA) where he is a categorical resident in General Surgery. He plans to work in the cardiac lab under Dr. Robbins during his research years. 